\title{
Correction to: Multi-Island Genetic-Algorithm-Based Approach to Uniquely Calibrate Polycrystal Plasticity Models for Magnesium Alloys
}

\author{
XIAOCHUAN SUN, ${ }^{1,2}$ BO ZHANG, ${ }^{3,6}$ YAODONG JIANG, ${ }^{4}$ \\ PEIDONG WU, ${ }^{5}$ and HUAMIAO WANG (10) ${ }^{1,2,7}$ \\ 1.-State Key Laboratory of Mechanical System and Vibration, Shanghai Jiao Tong \\ University, Shanghai 200240, China. 2.-Materials Genome Initiative Center, Shanghai Jiao \\ Tong University, Shanghai 200240, China. 3.-State Key Laboratory of Hydroscience and \\ Engineering, Tsinghua University, Beijing 100084, China. 4.-School of Mechanics \& Civil \\ Engineering, China University of Mining and Technology (Beijing), Beijing 100083, China. \\ 5.-Department of Mechanical Engineering, McMaster University, Hamilton, Ontario L8S \\ 4L7, Canada. 6.—e-mail: zbdth@mail.tsinghua.edu.cn. 7.—e-mail: wanghm02@sjtu.edu.cn
}

\section{Correction to:}

\section{JOM}

https://doi.org/10.1007/s11837-021-04614-0

This article was updated to correct duplication of author names in the reference lists.
Publisher's Note Springer Nature remains neutral with regard to jurisdictional claims in published maps and institutional affiliations. 